\title{
Effectiveness of Therapeutically Applied Behavior, Language and Fine Motor Occupational Therapy Sessions for Children with Autism Spectrum Disorder Aged 18 Months - 5 Years
}

\author{
Aastha Goel $^{1}$, Pooja Singh ${ }^{2}$, Malavika Samnani ${ }^{3}$ \\ ${ }^{1,2,3}$ Occupational Therapist, SOCH India, 483, Sec 27, Gurgaon , Haryana 122002, India
}

\begin{abstract}
Objective: To investigate that ABA based principles when applied in occupational therapy sessions can promote language comprehension, pre learning and pre-writing skills and behavior regulation in children with ASD. Method: A convenient sample of 20 children with provisional diagnosis of ASD were given occupational therapy sessions with specific goals post individual assessment of verbal language and fine motor skills .The goals were individualized as per their skill set and were based on a curriculum called ABLLS. The program was implemented at SOCH CENTRE for 45 minutes every day for duration of 2 months.The intervention used techniques like shaping, reinforcements, prompting and fading for behavior strategies.Fine motor activities targeted tripod grasp, in hand manipulations, translations and strengthening. The goal attainment scale was scored as followed- 0 for no, 1 for task with prompt and 2 for task accomplished in a child. Result: Inferential and descriptive statistics were used to analyze changes achieved over time and the change was statistically significant. Conclusion: Sessions focusing on fine motor skills can be used to improve visual tracking and visual attention, bilateral coordination, fine motor skills, verbal language and on task behaviors.
\end{abstract}

Keywords: applied behavior analysis, autism spectrum disorder, visual tracking, visual attention and bilateral co ordination.

\section{Introduction}

Children with ASDs exhibit qualitative impairments in reciprocal social interaction and in patterns of communication and demonstrate restricted, stereotyped and repetitive repertoires of interests and activities. These characteristics correspond to the triad of social interaction, communication and imagination impairments identified by Wing and Gould in 1979. It has been suggested that an additional dimension related to sensory perception might also be added to the triad (Autism Working Group, 2002a; Jones, 2002). The presence of these characteristics affects the manner in which children with ASDs interact with and understand the world.

Social impairments include an apparent unresponsiveness to other people, treating people or parts of people as inanimate objects, a lack of awareness of cultural norms or social perceptiveness, absence of empathy with the feelings of others, atypical use of eye contact, and an unawareness of the concept of "shared attention," which leads to joint referencing (Baron-Cohen and Bolton, 1993). Children with ASDs require direct teaching of social signals and conventions, such as responding to their name as an attention-alerting signal, turn-taking skills, the timing and dynamics of social interactions, the concept of sharing, the capacity to classify and respond to pertinent information, and the modulating of levels of arousal (Jordan, 2005).

Communicative impairments are characterized by an absence of meaningful communicative intent, difficulties in interpreting verbal and non-verbal expressions and gestures, confusion with the semantic and pragmatic aspects of language, speech patterns characterized by echolalia, metaphorical language, neologisms, and pronoun reversals
(Baron-Cohen and Bolton, 1993; Jordan and Powell, 1995). Jordan (1996) observes that ASDs are the only conditions where communication is separate from language in its development. Children with ASDs need to be directly taught the purpose of communication and the variety of ways in which we communicate, such as gestures, eye signaling, facial expression, and body posture (Jordan, 2005). The teaching of conversational skills related to turn-taking, active listening, topic introduction, maintenance and change will also form a central part of children's education programmers (Jordan, 2005). The literal understanding of children with ASDs presents particular difficulties, and a clear and unambiguous language of instruction is required in all learning and teaching situations.

Children with ASDs exhibit rigid thought and behavior patterns, which may lead to obsession behavior, repetitive interests.

\section{Aims And Objectives of the Study}

- To evaluate effect of applied behavior analysis based language and fine motor occupational therapy sessions with children with autism spectrum disorder.

- To adapt probe data sheets as a measure for evaluating pre learning, fine motor and behavior regulation skills.

\section{Hypothesis}

Null Hypothesis

- There is no significant effect of therapeutically applied behavior, language and fine motor occupational therapy sessions for children with autism spectrum disorder aged 18 months - 5 years.

- There is significant effect of therapeutically applied behavior, language and fine motor occupational therapy 


\section{International Journal of Science and Research (IJSR) \\ ISSN (Online): 2319-7064}

Index Copernicus Value (2013): 6.14 | Impact Factor (2014): 5.611

sessions for children with autism spectrum disorder aged 18 months -5 years.

\section{Methodology}

Research Design: Pre and post research design without control group .

Participation and study setting - Children between age group of 18months- 5 years with autism having language and communication, fine motor and behavioral difficulties were taken in one on one occupational therapy sessions in an inclusive settings at our centre. The rooms are enough to accommodate the children coming at different time intervals for occupational therapy sessions, like as 10 subjects in morning session and 10 in evening.

Techniques were incorporated from sensory integration, applied behavior analysis and behavior modification techniques.

Sampling: Convenient Sampling has been employed to involve the 20 subjects in the therapeutic program.

\section{Selection Criteria}

\section{Inclusion Criteria}

- Children falling under the age group between 18 months5 years.

- Children with both genders.

- Children with provisional diagnosis of Autism spectrum Disorder (ASD).

\section{Exclusion Criteria}

- High Modulation difficulties

- Emotionally deregulated children

\section{Variables}

- Independent - ABA based OT intervention

- Dependent - 1) fine motor skills 2) behavior 3) language and communication skills.

\section{Outcome Measures}

The categories were divided according to fine motor, behavior, language and communication based activities. The goal attainment scale was scored as followed- 0 for no, 1 for task with prompt and 2 for task accomplished in a child. Various sub domains like tripod grasp, in- hand manipulations, pretensions in fine motor skills, waiting concept in behavior skills and naming, labeling, receptive and expressive skills in language and communication skills broadly were taken in consideration.

\section{Intervention Specific Protocol}

One - on one language, behavior and fine motor sessions runs for time span of 45 mins everyday for two months. The ABA based OT sessions emphasized mainly of language communication and fine motor abilities.The intervention used techniques like shaping, reinforcements, prompting and fading for behavior strategies .Fine motor activities targeted tripod grasp, in-hand manipulations, translations and strengthening.

\section{Flow of Session and Handling}

1) In the intervention program, the therapist to child ratio was one to one and each child was supervised and trained individually targeting specific goals and activities.

2) The therapist provides reinforces which are part of protocol in form of bubbles, visual or sensory stuffs on task completion so that children do not exert counter control (tantrums and aggression), or become dependent on reinforces or prompts. Tangible re-inforcers were never given to ensure future endangering behavior that can occur and is unsafe for child.

3) Verbal and physical prompts were given to ensure effective learning of child.

4) To interpret behavior and communication skills, activities planned for sessions focused on visual performance, receptive language, imitation , requests and labels, intraverbals, fine motor

5) Concepts of waiting, instructor controlled structure , following one step or two step commands was encouraged to modify the target behavior thereby enhancing receptive and expressive skills also.

6) All the prompts especially for children who are nonverbal, pointing was judiciously used and gradually faded during course of program, hence promoting generalization, as based on ABA principles.

7) The intervention included fine motor tasks developing pincer grip including picking toothpicks, coins, develop in hand manipulations with tasks like placing pegs in pegboard using one hand only, removing and replacing screw lids, use of nuts and bolts, putting bindis in a shape drawn in front of child on a sheet, pulling string into the hand to make a string ball, play dough hand exercises for strengthening hand muscles (pounding and squashing)

8) Also enhancing tripod grasp by drawing and writing with crayon /chalks making standing and sleeping lines, pencil grippers. Holding a small jar or tube in the hand whilst trying to unscrew the lid with the same hand for the purpose of bilateral coordination.

\section{Results and Data Analysis}

Goal attainment scale was used, it was scored as followed- 0 for no, 1 for task with prompt and 2 for task accomplished in a child and probe data sheets that was used for scoring the goal or targets were marked daily by therapist. The results of all subjects under this study were evaluated pre and post intervention after two months by the therapists using above GAS.

The targets were divided broadly in categories of fine motor, receptive and expressive language skills , bilateral coordination, waiting concept , imitation , instruction following, joint attention .

The sub-categories under fine motor were tripod grasp, in hand manipulations, translations, pincer grasp etc. like holding a crayon or pencil with proper grasp while tracing or writing etc.

The receptive and expressive language whereas included pointing, labeling, instruction following using reinforcers, object, multiple actions with words like pick ball, pretend

\section{Volume 5 Issue 3, March 2016}




\section{International Journal of Science and Research (IJSR) \\ ISSN (Online): 2319-7064 \\ Index Copernicus Value (2013): 6.14 | Impact Factor (2014): 5.611}

action like laugh, yawn etc., locate body parts , use of intraverbals like fill in words from rhymes, animal sounds, naming fruits, answering multiple questions of one picture or object such as ball .

Instruction following without reinforcer was taken as separate domain, as task accomplishment itself was made as reinforcer with social gestures of high-5, high- 10 or clap.

In imitation vocal , oral, motor, were taken such as say moomoo, do clap clap, do action of rhyme such as twinkle twinkle, it was increased age appropriately as followed by one step to two step and more.

Bilateral coordination was targeted in each and every activity presented so as dominant hand was always encouraged to perform tasks while non- dominant supporting for holding and stabilization for. Ex while fixing pegs in magnetic peg board, or while weaving thread and needle in board or simple scissor cutting also eye hand coordination was included while performing this.

Lastly joint attention which is needed in every activity was also scored separately for each task so as to check if child is tracking for what's coming next or scanning or waiting for next command by therapist by giving eye gaze/ contact to activity as well as therapist simultaneously while performing tasks.

Table 1: Targets in Probe Data Sheet - Pre Intervention (Baseline Scores)

\begin{tabular}{|c|c|c|}
\hline Areas & Targets & $\begin{array}{c}\text { Target score at } \\
\text { base line }\end{array}$ \\
\hline Fine motor & 109 & 73 \\
\hline Receptive and expressive language skills & 265 & 111 \\
\hline Bilateral coordination & 71 & 39 \\
\hline Waiting concept & 208 & 161 \\
\hline Imitation & 102 & 56 \\
\hline Instruction following & 234 & 187 \\
\hline Joint attention & 160 & 125 \\
\hline
\end{tabular}

Table 2: Target In Probe Data Sheet - Post - Intervention (Scores Following Intervention)

\begin{tabular}{|c|c|c|}
\hline Areas & Targets & $\begin{array}{c}\text { Post } \\
\text { intervention } \\
\text { scores }\end{array}$ \\
\hline Fine motor & 109 & 87 \\
\hline Receptive and expressive language skills & 265 & 196 \\
\hline Bilateral coordination & 71 & 53 \\
\hline Waiting concept & 208 & 188 \\
\hline Imitation & 102 & 73 \\
\hline Instruction following & 234 & 208 \\
\hline Joint attention & 160 & 140 \\
\hline
\end{tabular}

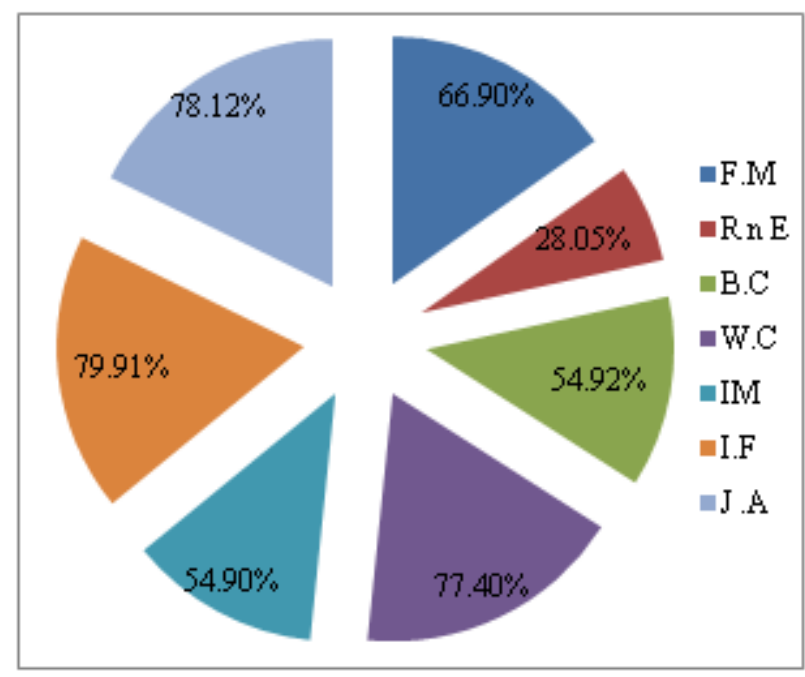

Graph 1: Pre-Intervention Scores

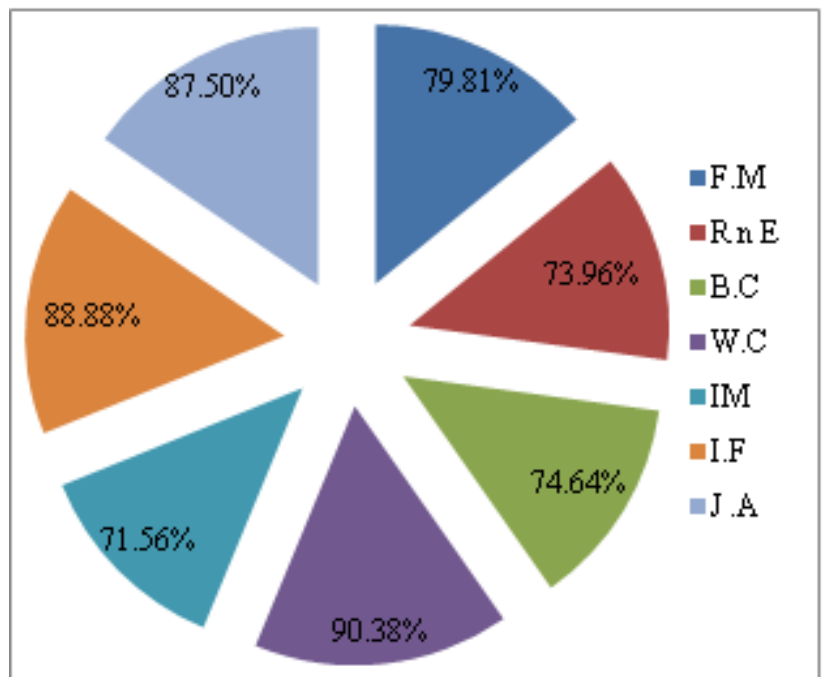

Graph 2: Post Intervention Scores

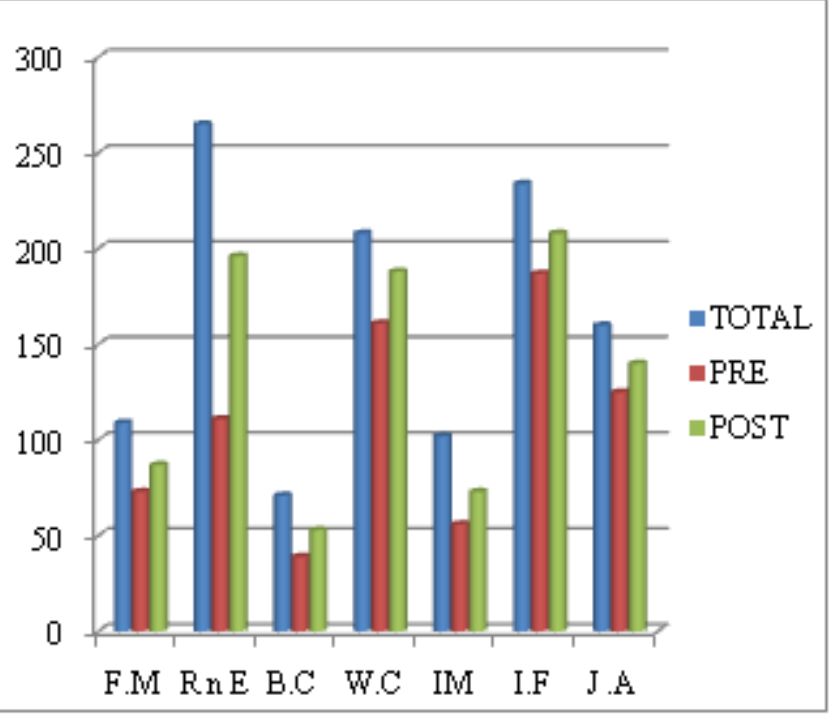

Graph 3: Comparative Scores of Pre Post and Total Targets in Probe Data Sheets

\section{Discussion}

The total targets taken for :Fine motor, imitation skills, waiting concept ,instruction following , bilateral coordination, receptive and expressive skills, joint attention 


\section{International Journal of Science and Research (IJSR) \\ ISSN (Online): 2319-7064 \\ Index Copernicus Value (2013): 6.14 | Impact Factor (2014): 5.611}

have shown significant improvement as compared and measured using GAS and probe sheets, as most goals or targets have been achieved in duration of two months like for receptive and expressive language using different strategies like incorporation of ABA, tracts, intraverbals, social skills for receptive and expressive skills, for fine motor incorporation of strengthening maneuvers like finger gripper, clay activities, in hand manipulations, bilateral coordination, Inclusion of waiting till 10 counts and gradually increasing counts helps in building waiting concept. Imitation that plays a key role by learning was incorporated through motor actions, vocal and oral, and for few subjects in our study where challenging behaviors of throwing, spitting , escaping ,attention seeking, shimming, mouthing were there, basic strategies underlying abc of behavior intervention were used thoroughly for those children . Challenging behavior though difficult to overcome in one - on one language sessions were taken into behavior modification or organization of behavior strategies using target behavior as a core area. Sandwich activities, proactive, environmental rearrangement etc. were used. As challenging behaviors takes time, so significant improvements can't be seen in shorter duration span, as intensive therapies and above mentioned strategies are used to overcome that.

\section{Limitations and Future Scope}

1) The research has a limitation of the small sample size taken, as the protocol adapted in one on one session at the centre has shown a significant trend of improvement in most of the domains and can be validated in near future studies with larger sample size.

2) As per data analyzed the fine motor ability have also shown changes post intervention for the children with ASD in small percentage thus a future research can concentrate to explore the fine motor tasks and activities in children with little and very poor fine motor functions.

3) The research study emphasizes on the challenging behavior for every child presently. In future study more than one challenging behavior can be brought to light and evaluated and intervened with the treatment protocol as adapted and validate its capability for standardization.

4) The research can explorate efficacy of combined effort of group and one on one session on language and behavior regulation in children with ASD predominantly showing modulation and emotional deregulation.

\section{References}

[1] American Psychiatric Association. (1994). Diagnostic and statistical manual of mental disorders (4th ed.). Washington, DC: Author.

[2] Anderson, A. E. (2002). Augmentative communication and autism: A com- parison of sign language and the picture exchange communication system (Doctoral dissertation, University of California at San Diego, 2001). Dissertation Abstracts International, 62, 4269-B.

[3] Anderson, A. E., \& Schreibman, L. (1999, May). Augmentative communication in autism: An exploration of characteristics that contribute to success. Paper presented at the Annual Meeting of the Association for Behavior Analysis, Chicago, IL.
[4] Anderson, S. R., Avery, D. L., DiPietro, E. K., Edwards, G. L., \& Christian, W. P. (1987). Intensive home-based early intervention with autistic children. Education \& Treatment of Children, 10, 352-366.

[5] Anderson, S. R., \& Romanczyk, R. G. (1999). Early intervention for young children with autism: Continuum-based behavioral models. Journal of the Association for Persons with Severe Handicaps, 24, 162-173. Bayley, N. (1993). Bayley scales of infant development (2nd ed.).

[6] San Antonio, TX: Psychological Corp. Bondy, A. S., \& Frost, L. A.(1994). The picture exchange communication system. Focus on Autistic Behavior, 9, $1-1$.

[7] Carr, E. G., Binkoff, J. A., Kologinsky, E., \& Eddy, M. (1978). Acquisition of sign language by autistic children: I. Expressive labeling. Journal of Applied Behavior Analysis, 11, 489-501.

[8] Charlop-Christy, M. H., Carpenter, M., Le, L., LeBlanc, L. A., \& Kellet, K. (2002). Using the Picture Exchange Communication System (PECS) with children with autism: Assessment of PECS acquisition, speech, socialcommunicative behavior, and problem behavior.

[9] Journal of Applied Behavior Analysis, 35, 213-231. Collaborative Workgroup on Autistic Spectrum Disorders. (1997). Best practices for designing and delivering effective programs for individuals with autistic spectrum disorders. Sacramento: California Department of Education.

[10] Frost, L., \& Bondy, A. (1994). The picture exchange communication system: Training manual. Newark, DE: Pyramid Consultants.

[11]Behavioral treatment and normal intellectual and educational functioning in autistic children. Journal of Consulting and Clinical Psychology, 55, 3-9.

[12] Grissmer, D. W., Grimm, K. J., Aiyer, S. M., Murrah, W. M., \& Steele, J. S. (2010). Fine motor skills and early comprehension of the world: Two new school readiness indicators.Developmental Psychology, 46, 1008-17. doi: 10.1037/a0020104. 